\title{
Chronic lower extremity wound infection due to Kerstersia gyiorum in a patient with Buerger's disease: a case report
}

\author{
Irmak Baran ${ }^{1,3^{*}} \mathbb{D}$, Arife Polat Düzgün², İpek Mumcuoğlu and Neriman Aksu'
}

\begin{abstract}
Background: Kerstersia gyiorum is an extremely rare pathogen of human infection. It can cause chronic infection in patients with underlying conditions. It can easily be misdiagnosed if proper diagnostic methods are not used.

Case presentation: A 47-year-old male patient with a history of Buerger's Disease for 28 years presented to our hospital with an infected chronic wound on foot. The wound was debrided, and the specimen was sent to Microbiology laboratory. Gram staining of the specimen showed abundant polymorphonuclear leukocytes and gram-negative bacilli. Four types of colonies were isolated on blood agar. These were identified as Kerstersia gyiorum, Proteus vulgaris, Enterobacter cloacae, Morganella morganii by Maldi Biotyper (Bruker Daltonics, Germany). The identification of $K$. gyiorum was confirmed by $16 \mathrm{~S}$ ribosomal RNA gene sequencing. The patient was successfully recovered with antimicrobial therapy, surgical debridement, and skin grafting.

Conclusions: This is the first case of wound infection due to K. gyiorum in a patient with Buerger's Disease. We made a brief review of $K$. gyiorum cases up to date. Also, this case is presented to draw attention to the use of new and advanced methods like MALDI-TOF MS and 165 rRNA gene sequencing for identification of rarely isolated species from clinical specimens of patients with chronic infections and with chronic underlying conditions.
\end{abstract}

Keywords: Kerstersia gyiorum, Buerger's Disease, Thromboangiitis obliterans, MALDI-TOF MS, 165 rRNA gene sequencing, Chronic wound infection

\section{Background}

Kerstersia gyiorum was first identified in 2003 by Coenye et al. as a distinct species by examination of the isolates obtained from nine clinical specimens such as leg ulcer, sputum, and faeces by cellular fatty acid analysis and 16S rRNA gene sequencing [1]. It belongs to Alcaligenaceae family and is closely related to Alcaligenes, Bordetella, Achromobacter spp. [2, 3]. After its first description, there have been publications reporting its isolation from chronic otitis media [2, 4-6], urinary tract infection [3], chronic leg ulcer [2], post-ulcer bacteraemia and sepsis [7] and bronchoalveolar lavage fluid [8].

\footnotetext{
* Correspondence: irmakmor@yahoo.com

${ }^{1}$ Medical Microbiology Department, Ankara Numune Research and Training

Hospital, Hacettepe Mahallesi Talatpasa Bulvari No: 44 Altindag, Ankara, Turkey

${ }^{3}$ Esat Caddesi 101/3 Kucukesat, 06660, Ankara, Turkey

Full list of author information is available at the end of the article
}

Here we present a case of chronic foot and ankle infection due to K. gyiorum in a 47-year-old patient with Buerger's Disease and we have made a brief review of $K$. gyiorum cases in literature so far.

\section{Case presentation}

A 47-year-old male patient who was previously followed up at the Chronic Wound Clinic of our hospital presented with a $10 \times 15 \mathrm{~cm}$ wound on the dorsolateral surface of the right foot and a $2 \times 3 \mathrm{~cm}$ wound on the outside of the right ankle (Figs. 1 and 2). The patient had a history of Buerger's Disease (Thromboangiitis obliterans) for 28 years. It has been learned that the patient continues smoking and lives in bad hygienic conditions. The patient received debridement of the wound at the Chronic Wound Clinic, and the sample was sent to our hospital's Medical Microbiology laboratory for microscopy and culture. Before getting culture results, oral 


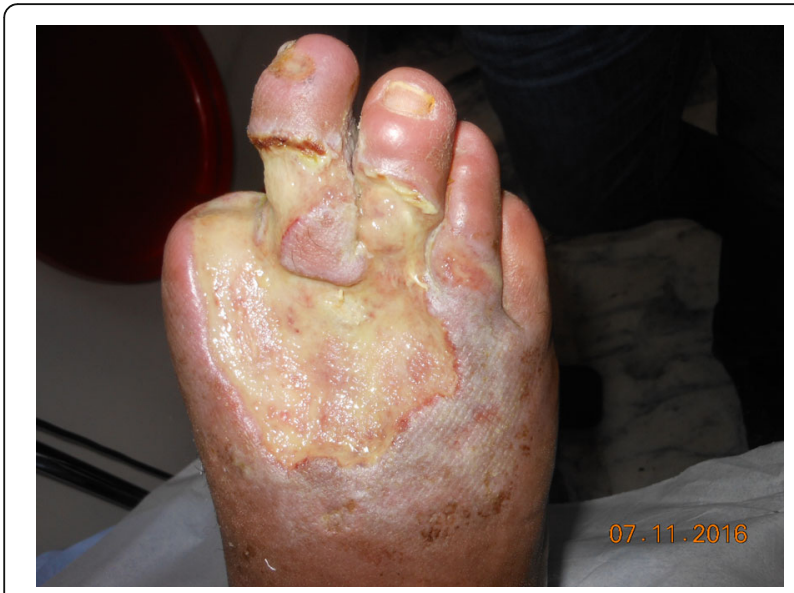

Fig. 1 Appearance of wound on the foot

ampicillin sulbactam, ciprofloxacin, and topical mupirocin treatment were started to the patient empirically.

When we questioned the patient he said that he had previously applied to the Chronic Wound Clinic nine months before this admission. The patient had first come to the Department of Cardiovascular Surgery because stem cell treatment was being considered. The patient had his toe amputated at another medical centre one month before that. The patient was referred to the Chronic Wound Clinic for the treatment of the infected chronic wound at his foot before stem cell treatment. In the blood tests performed at the first visit, the white blood cell count was $9500 / \mu \mathrm{L}$ (65.1\% neutrophils), hemoglobin $12.3 \mathrm{~g} / \mathrm{dl}$, hematocrit $38.9 \%$, platelets $348.000 / \mu \mathrm{L}$. Both the patient's fasting blood glucose (95 mg/dL) and \% HbA1c (6.1\%) levels were found to be normal. The patient had higher than normal levels of CRP $(12 \mathrm{mg} / \mathrm{L})$ and erythrocyte sedimentation rate (55 mm/h). The patient's coagulation tests were within normal limits (aPTT $31.8 \mathrm{~s}$, prothrombin time $11 \mathrm{~s}$, INR

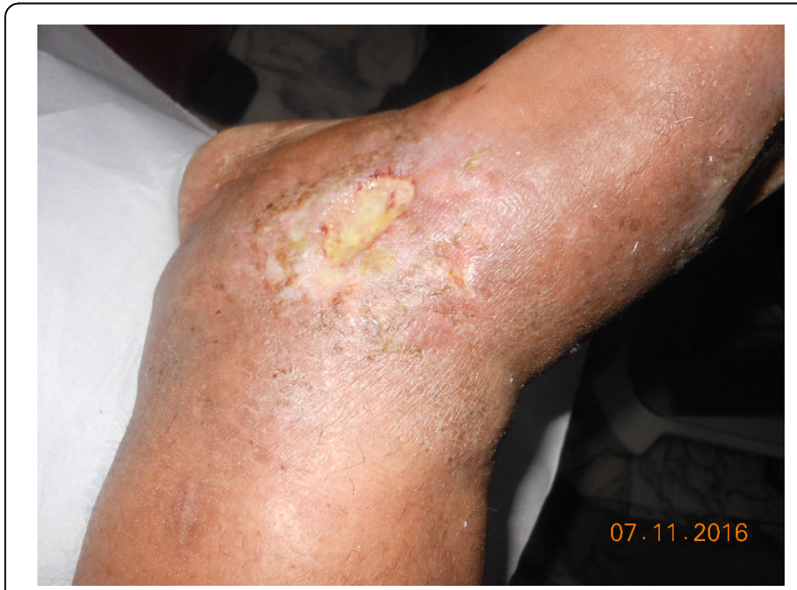

Fig. 2 Appearance of wound on ankle
0.99). The patient's HBsAg, Anti-HIV, and Anti-HCV tests were shown to be negative with ELISA method (ETI-MAX 3000 analyzer; DiaSorin S.p.A., UK). The angiography of the leg showed left deep femoral artery had thin calibration, the right superficial femoral artery was occluded at the level of the $1 / 2$ middle of the thigh, and there were intense collateral arteries on leg and thigh. Microscopic examination of the specimen taken from the wound revealed 4-5 polymorphonuclear leucocytes in each area. Pseudomonas aeruginosa was isolated from wound culture. When antibiotic sensitivity tests were made this microorganism was found to be sensitive to tobramycin, colistin, ceftazidime, and gentamicin. The patient was given iv ceftazidime and metronidazole treatment for 14 days. The patient was hospitalized for one month, and treated with hyperbaric oxygen therapy. After skin grafting, the patient was discharged.

This time the previous graft was found to be lysed, and the wound was re-infected. The blood tests were performed and white blood cells were found $8200 / \mu \mathrm{L}$ (70\% neutrophils), hemoglobin $14 \mathrm{~g} / \mathrm{dl}$, hematocrit $43.7 \%$, and platelets $306.000 / \mu \mathrm{L}$. The patient's CRP $(7 \mathrm{mg} / \mathrm{L})$ and erythrocyte sedimentation rate $(65 \mathrm{~mm} /$ h) were found to be high.

Plenty of polymorphonuclear leukocytes and gramnegative bacilli were present in the Gram stained sample taken from the patient's wound which was sent to our hospital's Medical Microbiology laboratory. The material was inoculated on to blood agar and Eosin methylene blue (EMB) agar and incubated. Four different types of colonies were identified after $24 \mathrm{~h}$ of incubation at $37^{\circ} \mathrm{C}$ on bloody agar and EMB agar. Out of these four colony types, the colonies predominantly grown on blood agar were light gray, had a dry appearance, and were large colonies with indented edges that tended to merge with each other, resembling Alcaligenes. However, unlike Alcaligenes there was no fruity odour and oxidase test

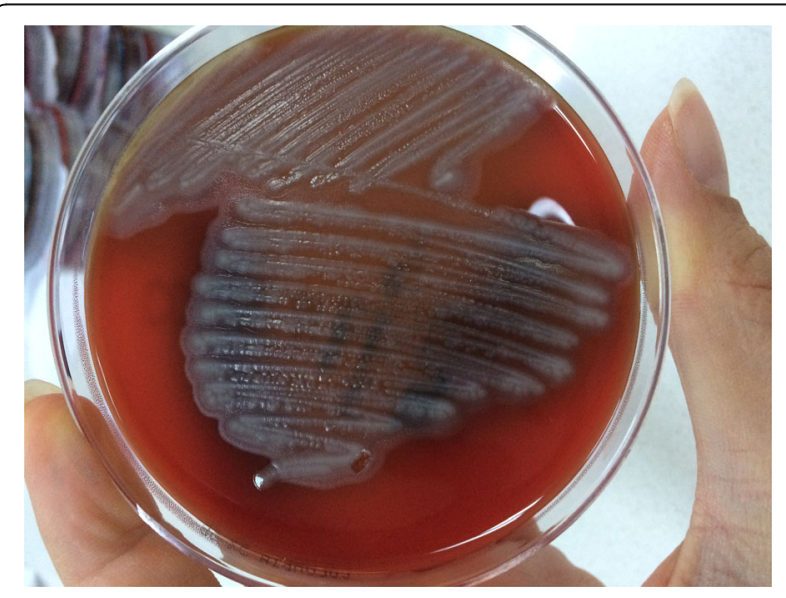

Fig. 3 Kerstersia gyiorum on blood agar 


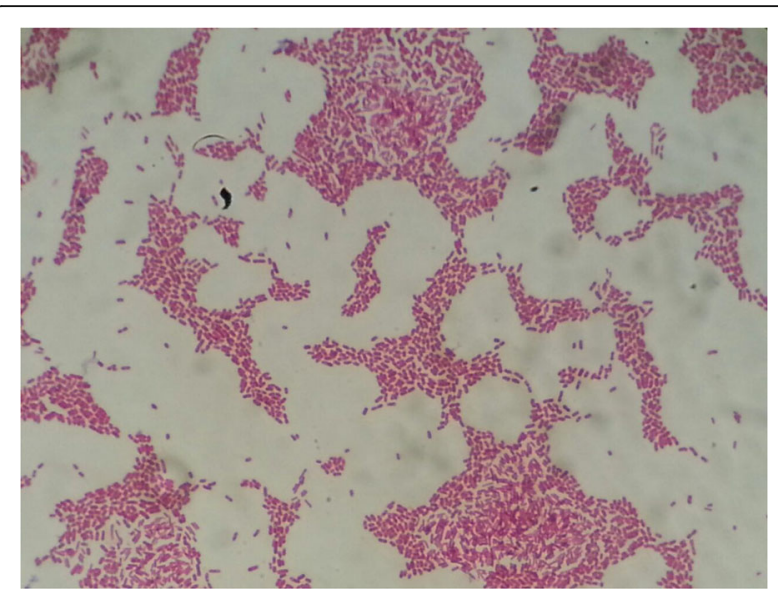

Fig. 4 Gram stain from the pure culture of Kerstersia gyiorum. Gramnegative, short rods were seen

was negative. These colonies were found to be indolenegative, urease-negative and strongly catalase-positive. The colonies grown on blood agar with second density were gray coloured and had swarming around the colony, were indole positive, urease positive, oxidase negative, hydrogen sulphide producing colonies. The third colony type was dirty-white-coloured, mucoid with smooth edges. They were indole negative, VogesProskauer test positive. The fourth colony type was smooth-edged, whitish mucoid colonies. Urease test was positive, indole positive and Voges-Proskauer test was negative. On EMB medium a mixed growth of one lactose-positive and three lactose-negative colonies corresponding to these four types of colonies was observed. Single colony passages were made from each of these four colony types in order to identify with Maldi
Biotyper (Bruker Daltonics, Germany) system, a matrixassisted laser desorption ionization-time of flight mass spectrometry (MALDI-TOF MS) system in our Medical Microbiology Laboratory. Antibiotic susceptibility tests of the bacteria were carried out on a Phoenix 100 (Beckton Dickinson, USA) device.

The passages made from the first colony type produced dry, light gray, opaque colonies, with irregularly indented, protruding edges, and swarming around the colonies on blood agar (Fig. 3), and lactose-negative colonies on EMB. Gram-negative, short bacilli were seen in Gram staining of colonies from blood agar (Fig. 4). These colonies were identified as Kerstersia gyiorum by Maldi Biotyper (Bruker Daltonics, Germany) system with 2.348 Biotyper score (excellent identification). The identification of $K$. gyiorum with Maldi Biotyper was confirmed by $16 \mathrm{~S}$ ribosomal RNA gene sequencing according to the predefined methodology $[9,10]$. Briefly, the following steps were taken for this procedure. DNA was isolated from pure culture using DNeasy Blood \& tissue kit (Qiagen, Germany). 16S rRNA gene amplification was performed using universal 27F (AGAGTTT GATCMTGGCTCAG) and 1492R (GGTTACCTTGT TACGACTT) primers. In the PCR reaction, 1XTaq buffer, $2 \mathrm{mM} \mathrm{MgCl} 2,0.2 \mathrm{mM} \mathrm{dNTP}, 0.4$ pmol of primers and 1.25 U Taq polymerase (Thermo Fisher Scientific, USA) were used in a volume of $50 \mu \mathrm{L}$. DNA sequencing of the resulting product was performed using the Bigdye Cycle Sequencing Kit v.3.1 (Applied Biosystems, USA). Sequence analyzes were compared to the GenBank NCBI gene library data using the BLAST program (https://blast.ncbi.nlm.nih.gov/Blast.cgi). The sequence was found $100 \%$ identical to K. gyiorum. Antibiotic susceptibility tests for $K$. gyiorum were performed using the Phoenix 100

Table 1 Antimicrobial susceptibility profile of Kerstersia gyiorum as determined by Phoenix 100 and E-test

\begin{tabular}{lllll}
\hline & Phoenix 100 & & E-test \\
\hline Antimicrobial agent & MIC $(\mu \mathrm{g} / \mathrm{mL})$ & Result & MIC $(\mu \mathrm{g} / \mathrm{mL})$ & - \\
Amikacin & $\leq 4$ & Susceptible & - & Result \\
Aztreonam & $\leq 1$ & Susceptible & - & - \\
Ceftazidime & $\leq 0.5$ & Susceptible & -25 & - \\
Ceftriaxone & - & - & 0.25 & Susceptible \\
Ciprofloxacin & $\leq 0.125$ & Susceptible & Susceptible \\
Colistin & $>4$ & Resistant & 1 & Resistant \\
Gentamicin & 2 & Susceptible & - & Susceptible \\
Imipenem & - & - & Susceptible \\
Meropenem & $\leq 0.125$ & Susceptible & - & - \\
Netilmicin & 2 & Susceptible & - & - \\
Piperacillin & $\leq 4$ & Susceptible & - & - \\
Piperacillin-tazobactam & $\leq 4 / 4$ & Susceptible & - & Susceptible \\
Trimethoprim-sulfamethoxazole & $\leq 1 / 19$ & Susceptible & $\leq 2 / 38$ & \\
\hline
\end{tabular}


(Beckton Dickinson, USA) device and the E-test (Liofilchem, Italy) for six antimicrobial agents (ceftriaxone, ciprofloxacin, colistin, gentamicin, imipenem, trimethoprimsulfamethoxazole) on Mueller-Hinton agar in accordance with the manufacturer's specifications. The results were evaluated according to the MIC breakpoints established by Clinical and Laboratory Standards Institute for other nonEnterobacteriaceae [11]. The results of antibiotic susceptibility tests for $K$. gyiorum are shown in Table 1 .

Other microorganisms were identified as Proteus vulgaris, Enterobacter cloacae, Morganella morganii by Maldi Biotyper system, respectively. Antibiotic susceptibility results of $P$. vulgaris, E. cloacae, M. morganii are shown in Table 2.

Oral ampicillin-sulbactam and ciprofloxacin treatment was continued because the microorganisms were susceptible to these antibiotics. Hyperbaric oxygen therapy was used. Wound debridement and skin grafting were applied. The patient was discharged after one month of hospitalization. The patient was doing well in follow-up examination three-months later (Fig. 5). Figure 6 shows a timeline of events.

\section{Discussion}

K. gyiorum's name was derived from the Greek word "gyion", which means "limb" since it was often isolated from leg and ankle wounds when it was first described

Table 2 Antimicrobial susceptibility results of Proteus vulgaris, Enterobacter cloacae, Morganella morganii

\begin{tabular}{|c|c|c|c|}
\hline & $\begin{array}{l}\text { Proteus } \\
\text { vulgaris }\end{array}$ & $\begin{array}{l}\text { Enterobacter } \\
\text { cloacae }\end{array}$ & $\begin{array}{l}\text { Morganella } \\
\text { morganii }\end{array}$ \\
\hline Amikacin & Susceptible & Susceptible & Susceptible \\
\hline Amoxicillin-clavulanic acid & Susceptible & Resistant & Resistant \\
\hline Ampicillin & Resistant & Resistant & Resistant \\
\hline Ampicillin-sulbactam & Susceptible & Susceptible & Susceptible \\
\hline Ertapenem & Susceptible & Susceptible & Susceptible \\
\hline Gentamicin & Susceptible & Susceptible & Susceptible \\
\hline Imipenem & Intermediate & Susceptible & Susceptible \\
\hline Levofloxacin & Susceptible & Susceptible & Susceptible \\
\hline Meropenem & Susceptible & Susceptible & Susceptible \\
\hline Piperacillin-tazobactam & Susceptible & Susceptible & Susceptible \\
\hline Cefepime & Susceptible & Susceptible & Susceptible \\
\hline Cefoxitin & Susceptible & Susceptible & Susceptible \\
\hline Cefotaxime & Susceptible & Susceptible & Susceptible \\
\hline Ceftriaxone & Resistant & Susceptible & Susceptible \\
\hline Cefuroxime & Susceptible & Susceptible & Susceptible \\
\hline Ciprofloxacin & Susceptible & Susceptible & Susceptible \\
\hline Tobramycin & Susceptible & Susceptible & Susceptible \\
\hline $\begin{array}{l}\text { Trimethoprim- } \\
\text { sulfamethoxazole }\end{array}$ & Resistant & Resistant & Susceptible \\
\hline
\end{tabular}

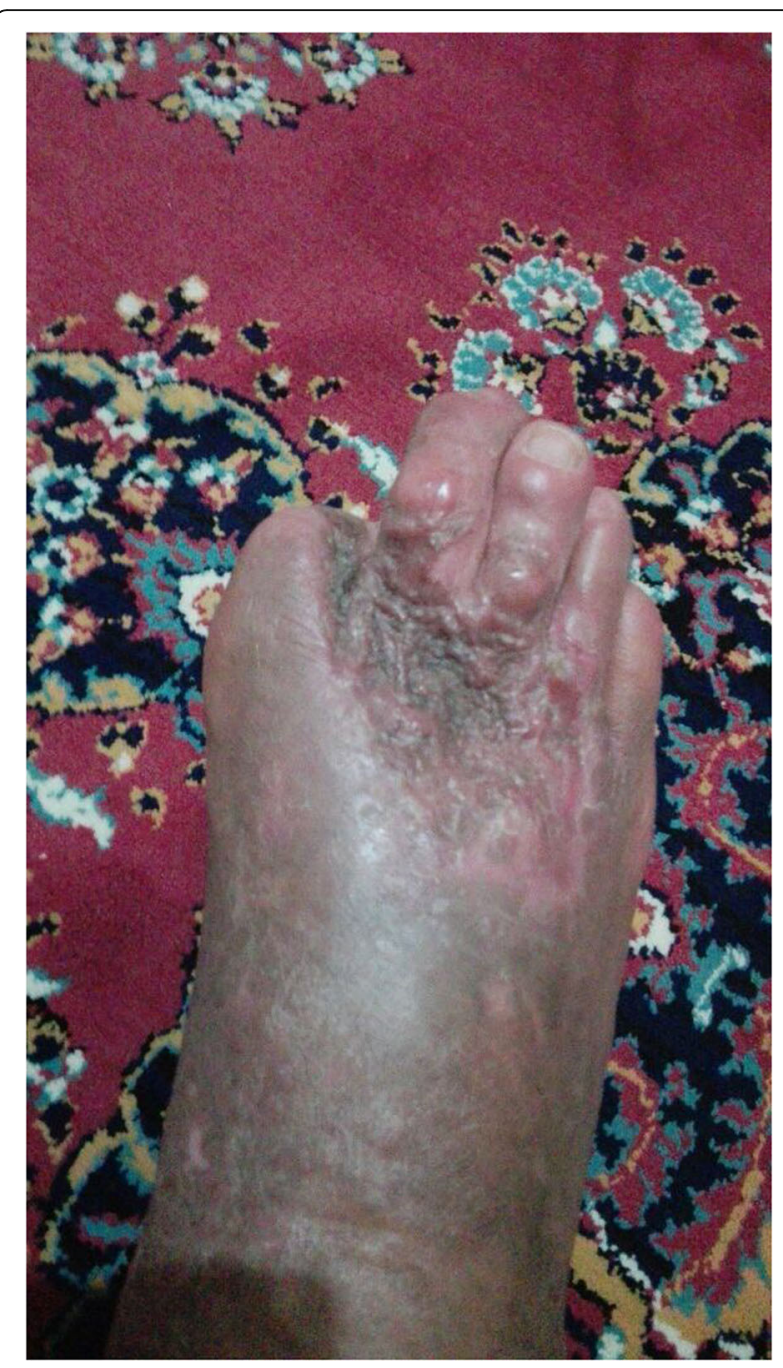

Fig. 5 Appearance of the patient's foot at the three-month follow-up

[1-3, 6, 7]. K. gyiorum belongs to Alcaligenaceae family, and it is related to Alcaligenes, Bordatella, Achromobacter, and Pigmentiphaga generas [1, 3-7]. K. gyiorum colonies are known to show an appearance similar to Alcaligenes faecalis. However, K. gyiorum isolates are oxidase negative and lack the characteristic fruity odor $[3,7]$. $K$. gyiorum is highly catalase positive but urease and $\beta$-galactosidase negative [1].

When we conducted a search for "Kerstersia gyiorum case report" on PubMed and Medline we found that there were 9 cases since the first time it was defined in 2003 [2-8] Table 3 shows clinical features of these case reports. In 2012, Vandamme et al. identified Kerstersia similis, a close species, from the neck abscess of a 54year-old patient [12].

In our case $K$. gyiorum grew with other types of microorganisms from wound specimen. This was also observed by previous researchers who have reported cases of $K$. gyiorum [2-8]. In seven of the nine cases that have 


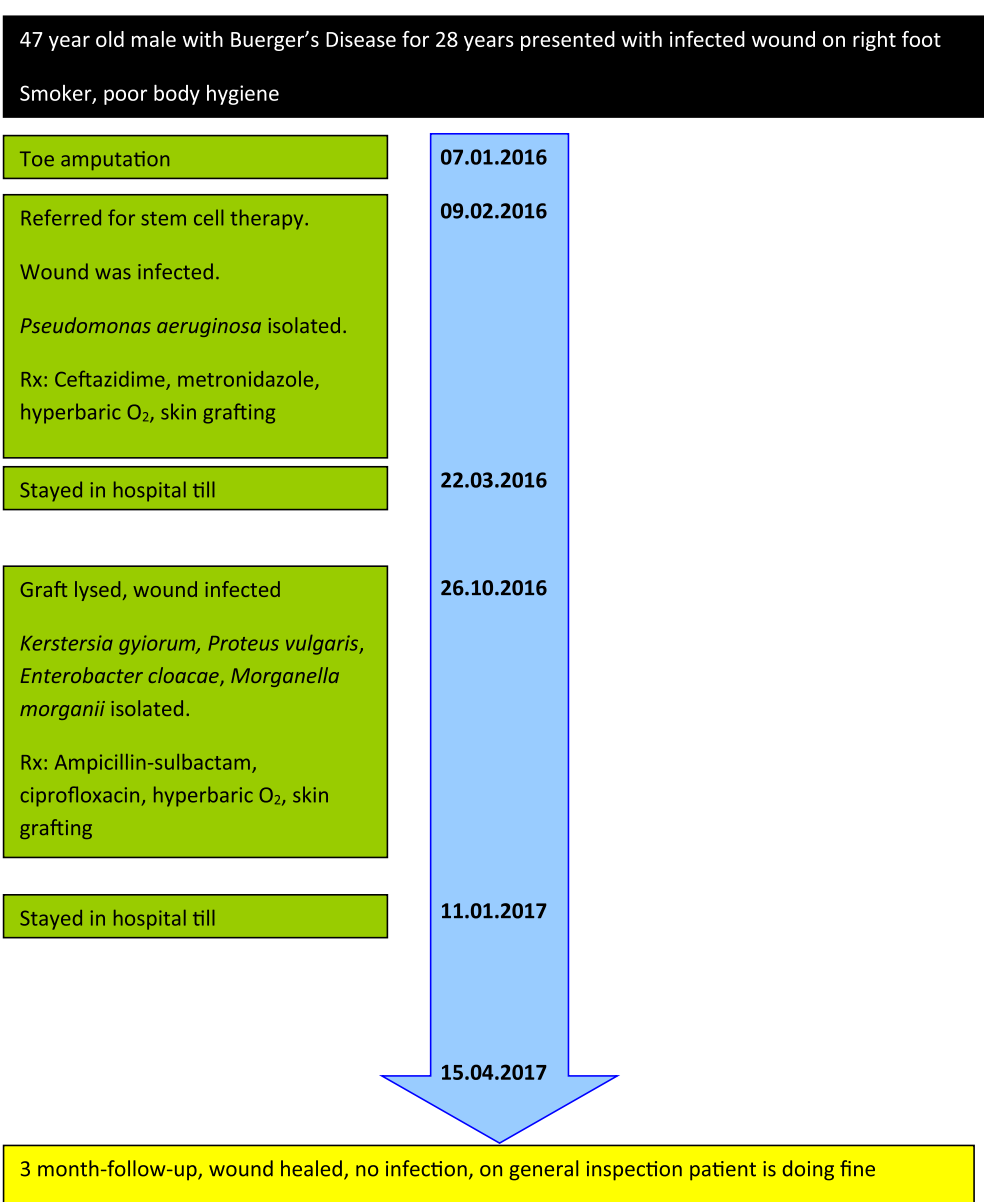

Fig. 6 A timeline of all events since patient's first hospitalization

been reported so far there were polymicrobial infections. $K$ gyiorum was isolated from urinary tract infection together with $P$. vulgaris [3], from chronic otitis media with Corynebacterium amycolatum, from chronic lower extremity wound with Morganella spp. [2], from bronchoalveolar lavage fluid with Pseudomonas aeruginosa and Stenotrophomonas maltophilia [8], from chronic suppurative otitis media with $P$. aeruginosa in a case report [5] from our country, and in two different cases from chronic suppurative otitis media with Proteus mirabilis and from chronic suppurative otitis media with Staphylococcus aureus and Escherichia coli [6]. In our case, $K$. gyiorum was seen to be grown more dominantly than other microorganisms in the culture plate. However, since there are a very limited number of reports in the literature on this microorganism, it is difficult to predict the effect of $K$. gyiorum in the disease process in a polymicrobial infection [2]. The virulence factors of this microorganism should be investigated [6]. In our case, the patient's condition got better after antimicrobial therapy. Ogawa et al. reported that $K$. gyiorum is prone to cause infection in situations predisposing to polymicrobial infection because Achromobacter and Alcaligenes spp. which are closely related have the same tendency [3].

If we look at the results of antibiotic susceptibility, our K. gyiorum isolate was found to be susceptible to aminoglycosides, ciprofloxacin, imipenem and meropenem and broad spectrum cephalosporins. These results were similar to Coenye et al. [1] and Almuzara et al.'s results [4]. However, Pence et al. [2], Uysal et al. [5], Mwalutende et al. [6], Bostwick et al. [7], Deutscher et al. [8], found resistance to ciprofloxacin in $K$. gyiorum isolates.

Our patient was a smoker. He had Buerger's Disease (Thromboangiitis obliterans) for 28 years due to smoking. Thromboangiitis obliterans is a non-atherosclerotic inflammatory disease affecting the small and medium vessels in the limbs and causes circulatory problems [13]. In this disease, ulcers that do not heal especially at the distal end of the extremities are seen $[13,14]$. The chronic suppurative otitis media case due to $K$. gyiorum that Mwalutende et al. reported was a chronic smoker [6]. Pence et al. also reported chronic cigarette smoking in their case [2]. We believe that there may be a 


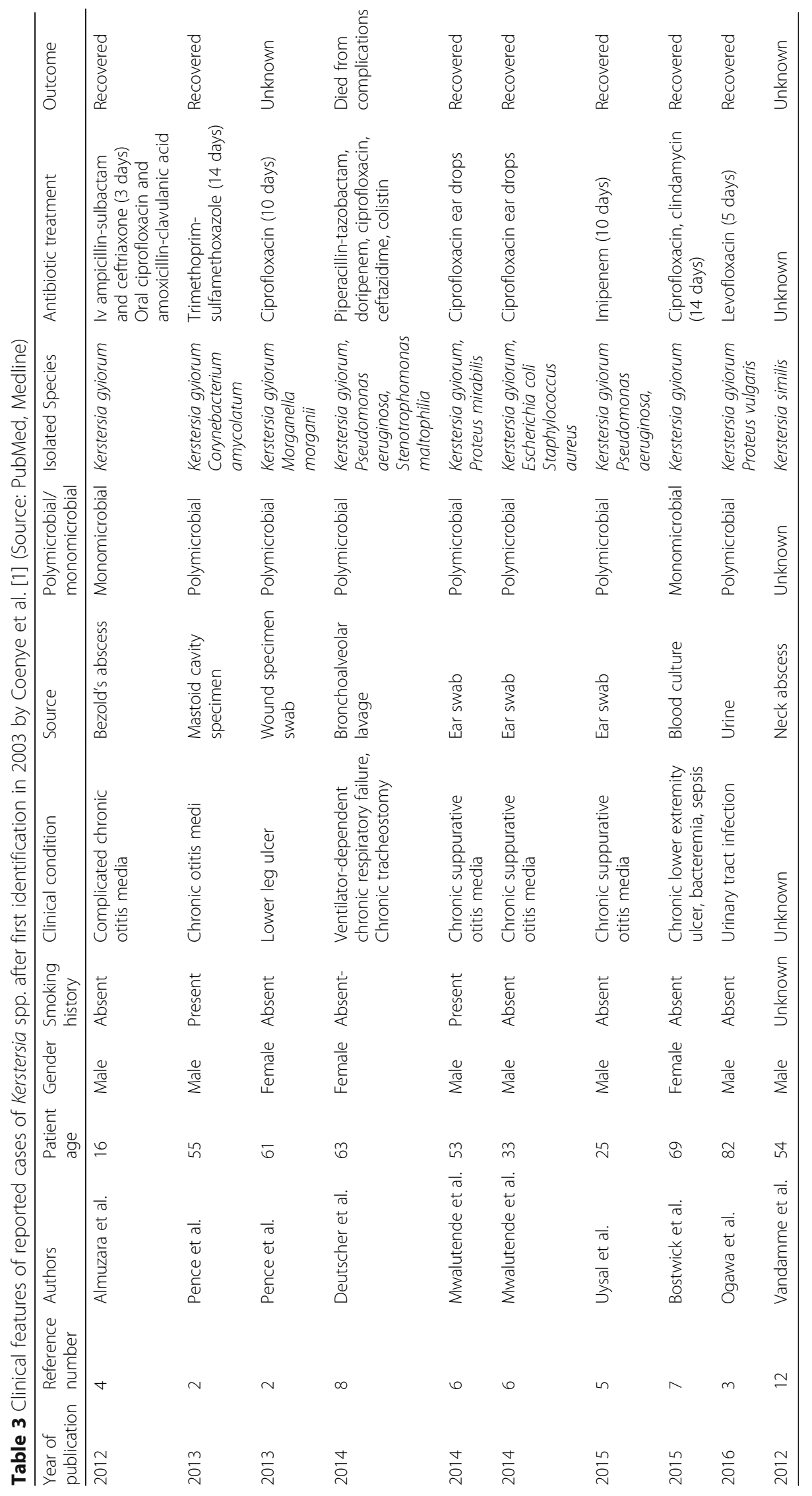


relationship between chronic smoking and infection due to $K$. gyiorum. However, further work is needed in order to test this hypothesis.

Previous researchers who reported case reports of $K$. gyiorum reported that the associated infection usually develops on a long-standing inflammatory condition $[2-4,6,8]$. Our patient had long-term lower extremity ulcers due to Buerger's Disease.

In our case, we observed that on blood agar $K$. gyiorum forms colonies with a irregular spreading edge morphology. Pence et al., Deutscher et al., and Bostwick et al. reported that they observed colonies with similar appearance to ours $[2,7,8]$. We think that these phenotypical features may be typical for $K$. gyiorum. This morphology can be used to distinguish $K$. gyiorum from Acinetobacter spp., which is also a nonfermentative and oxidase-negative bacterium as suggested by previous researchers $[2,8]$. Pence et al. referred to the formation of lavender pigment on MacConkey agar [2]. We did not see such pigment formation in our own case. However we used EMB instead of MacConkey agar. In our opinion, the appearance of such a colony type after inoculation of a specimen from a site of chronic inflammation should suggest $K$. gyiorum infection.

Introduction of molecular and genetic identification methods to clinical microbiology has increased the detection of new and rare bacteria in clinical specimens. MALDI-TOF MS and 16S rRNA gene sequencing are two of these new and advanced methods $[2,6,8]$. In our case we detected the presence of $K$. gyiorum by using MALDI-TOF MS. The reliance on solely biochemical identification methods or automated identification systems may lead to misidentification of $K$. gyiorum, because it has common biochemical features with more commonly detected pathogens such as Acinetobacter spp. [5-7]. Also $K$. gyiorum is phenotypically similar to Alcaligenes faecalis and it may go unnoticed if proper identification methods are not used [5]. The increase in the identification of new and previously unrecognized bacteria has led to two important situations in clinical microbiology laboratories. The first is to determine whether these microorganisms are pathogens or contaminants, and the second is to correctly determine the antibiotic susceptibilities of these microorganisms [2]. The co-operation of the clinician and the laboratory is required to achieve these. As the data in the literature will increase with the studies to be done on this subject, a more accurate perspective will be obtained for approaching these cases. Besides, it is difficult to identify such rare microorganisms by conventional methods. Therefore, when the pathogen cannot be identified by routine biochemical methods in cases of chronic infections that do not heal, MALDI-TOF MS and 16S rRNA gene sequencing are emerging as fast and reliable alternatives $[5,8]$.

\section{Conclusions}

We think that $K$. gyiorum should be kept in mind as a possible agent associated with chronic infected lower extremity ulcers in patients with Buerger's Disease.

\section{Abbreviations \\ 16S rRNA: 16 S ribosomal ribonucleic acid; Anti-HCV: Anti hepatitis C virus antibodies; Anti-HIV: Anti human immunodeficiency virus antibodies; aPTT: Activated partial thromboplastin time; CRP: C-reactive protein; ELISA: Enzyme-linked immunosorbent assay; EMB: Eosin methylene blue; E- test: Epsilometer test; HbA1c: Glycated haemoglobin; HBsAg: Surface antigen of the hepatitis B virus; INR: International normalized ratio; MALDI-TOF MS: Matrix-assisted laser desorption ionization-time of flight mass spectrom- etry; MIC: Minimum inhibitory concentration; NCBI: National Center for Biotechnology Information; PCR: Polymerase chain reaction}

\section{Acknowledgements}

The authors wish to acknowledge the patient.

Funding

The authors declare that funding was not utilized for the preparation of this manuscript.

Availability of data and materials

All data generated or analysed during this study are included in this published article.

\section{Authors' contributions}

IB isolated the microorganisms, did antimicrobial susceptibility tests and molecular tests, conducted the literature review, collected the data from the patient's medical records and wrote the manuscript. APD followed the patient, made surgical wound debridement and skin grafting, prospectively recorded the patient's clinical data, and took the pictures of the patient. IM conducted E-tests, involved in analysis of data and drafting of the manuscript. NA involved in supervision of the laboratory tests and revised crucially the manuscript for important intellectual content. All authors read and approved the final manuscript.

Ethics approval and consent to participate

Our ethics committee waived the requirement of ethics approval because all medical and laboratory procedures are routinely carried out and do not affect decisions concerning treatment.

\section{Consent for publication}

Written informed consent was obtained from the patient for publication of this case report. A copy of the written consent is available for review by the Editor of this journal.

\section{Competing interests}

The authors declare that they have no competing interests.

\section{Publisher's Note}

Springer Nature remains neutral with regard to jurisdictional claims in published maps and institutional affiliations.

\section{Author details}

${ }^{1}$ Medical Microbiology Department, Ankara Numune Research and Training Hospital, Hacettepe Mahallesi Talatpasa Bulvari No: 44 Altindag, Ankara, Turkey. ${ }^{2}$ Clinic of General Surgery, Ankara Numune Research and Training Hospital, Ankara, Turkey. ${ }^{3}$ Esat Caddesi 101/3 Kucukesat, 06660, Ankara, Turkey. 
Received: 27 April 2017 Accepted: 31 August 2017

\section{Published online: 06 September 2017}

\section{References}

1. Coenye T, Vancanneyt M, Cnockaert MC, Falsen E, Swings J, Vandamme P. Kerstersia gyiorum gen. nov., sp. nov., a novel Alcaligenes faecalis-like organism isolated from human clinical samples, and reclassification of Alcaligenes denitrificans Rüger and Tan 1983 as Achromobacter denitrificans comb. nov. Int J Syst Evol Microbiol. 2003;53(Pt 6)::1825-31.

2. Pence MA, Sharon J, McElvania Tekippe E, Pakalniskis BL, Ford BA, Burnham CA. Two cases of Kerstersia gyiorum isolated from sites of chronic infection. J Clin Microbiol. 2013;51(6):2001-4.

3. Ogawa Y, Lee ST, Kasahara K, Koizumi A, Chihara Y, Nakano R, Yano H, Mikasa K. A first case of isolation of Kerstersia gyiorum from urinary tract. J Infect Chemother. 2016;22(4):265-7.

4. Almuzara MN, Barberis CM, Traglia GM, Ordoñez AM, Famiglietti AM, Ramirez MS, Vay CA. Isolation of Kerstersia gyiorum from a patient with cholesteatomatous chronic otitis media. J Clin Microbiol. 2012;50(11):3809-11.

5. Uysal EB, Çelik C, Tuzcu N, Can F, Doğan M, Ertürk R, Bakici MZ. A case of chronic suppurative otitis media caused by Kerstersia gyiorum. APMIS. 2015; 123(11):986-9.

6. Mwalutende A, Mshana SE, Mirambo MM, Mushi MF, Chalya PL, Giliyoma JM, Schneiderhan W, Zimmermann O, Groß U. Two cases of chronic suppurative otitis media caused by Kerstersia gyiorum in Tanzania: is it an underappreciated pathogen in chronic otitis media? Int J Infect Dis. 2014;29: 251-3.

7. Bostwick AD, Zhang C, Manninen K, Touchberry J, Greene SR, Holland TL. Bacteremia caused by Kerstersia gyiorum. J Clin Microbiol. 2015;53(6):1965-7.

8. Deutscher M, Severing J, Balada-Llasat JM. Kerstersia gyiorum isolated from a Bronchoalveolar lavage in a patient with a chronic tracheostomy. Case Rep Infect Dis. 2014;2014:479581.

9. Watson ME Jr, Estabrook MM, Burnham CA. Catheter-associated Nocardia Higoensis bacteremia in a child with acute lymphocytic leukemia. J Clin Microbiol. 2011:49(1):469-71.

10. Ziga ED, Druley T, Burnham CA. Herbaspirillum species bacteremia in a pediatric oncology patient. J Clin Microbiol. 2010;48(11):4320-1.

11. Clinical and Laboratory Standards Institute (CLSI). Performance Standards for Antimicrobial Susceptibility Testing; Twenty-forth Informational Supplement. M100-S24. Wayne, PA, USA: 2014

12. Vandamme $P$, De Brandt $E$, Houf $K$, De Baere T. Kerstersia similis sp. nov. isolated from human clinical samples. Int I Syst Evol Microbiol. 2012;62(Pt 9): 2156-9.

13. Rivera-Chavarría IJ, Brenes-Gutiérrez JD. Thromboangiitis obliterans (Buerger's disease). Ann Med Surg (Lond). 2016;7:79-82.

14. Piazza G, Creager MA. Thromboangiitis obliterans. Circulation. 2010;121(16): 1858-61.

\section{Submit your next manuscript to BioMed Central and we will help you at every step:}

- We accept pre-submission inquiries

- Our selector tool helps you to find the most relevant journal

- We provide round the clock customer support

- Convenient online submission

- Thorough peer review

- Inclusion in PubMed and all major indexing services

- Maximum visibility for your research

Submit your manuscript at www.biomedcentral.com/submit 